\title{
La Problematique De Gestion Du Parc National De La Comoe (Pnc) En Cote D'ivoire, Entre La Survie Des Populations Riveraines Et La Conservation De La Biodiversite
}

\author{
Koueita Mariam Konaté Kady \\ Diomandé Moussa \\ Brou AhossiNicolas
}

Institut des Sciences Anthropologiques de Développement

Université Félix Houphouët-Boigny (Cocody-Abidjan), Côte d'Ivoire

Doi:10.19044/esj.2018.v14n35p391 URL:http://dx.doi.org/10.19044/esj.2018.v14n35p391

\begin{abstract}
The Comoé National Park (CNP), the largest park in West Africa, is one of the twenty largest parks in the world. It undergoes many anthropics pressures, the most intense of its history have been those periods of sociopolitical crisis that Côte d'Ivoire has experienced. The anthropogenic pressures that weigh on this park are most often practiced by the riparian populations for their survival. These pressures are rife despite the State's investigations through the Ivorian Office of Parks and Reserves (OIPR), the structure in charge of the management of protected areas. In view of this situation, a fundamental question arises, how to achieve sustainable conservation of the park's biodiversity without depriving riparian populations of their survival? This study mainly allows to analyze the factors at the origin of the management problem of this park. Through a qualitative approach as well as the appropriate data collection techniques and tools, we identify the problems from three main angles, namely the socio-cultural, economic and institutional characteristics that underlie the sustainable management problem of the CNP.
\end{abstract}

Keywords: Biodiversity, conservation, protected area, riparian population, survival

\section{Résumé}

Le Parc National de la Comoé (PNC), le plus grand parc de l'Afrique de l'Ouest, fait partie des vingt plus grands parcs du monde. Il subit de nombreuses pressions anthropiques dont les plus intenses de son histoire ont été celles des périodes de crise sociopolitique que la Côte d'Ivoire a connue. 
Les pressions anthropiques qui pèsent sur ce parc,sontle plus souvent pratiquées par les populations riveraines pour leur survie. Ces pressions sévissent en dépit des investigations de l'Etat à travers l'Office Ivoirien des Parcs et réserves (OIPR),la structure en charge de la gestion des aires protégées. Au regard de cette situation, une question fondamentale se pose, comment parvenir à conserver durablement la biodiversité du parc sans toutefois priver les populations riveraines de leur survie ? Cette étude permet principalement d'analyser les facteurs à l'origine de la problématique de gestion de ce parc. A travers une approche qualitative ainsi que les techniques et outils de collecte de données appropriés, nous identifions les problèmes sous trois principaux angles à savoir, les caractéristiques socioculturelle, économique et institutionnelle à la base de la problématique de gestion durable du PNC.

Mots clés : Aire protégée, biodiversité; conservation, population riveraine; survie

\section{INTRODUCTION}

Les aires protégées ont été créées afin de palier à la dégradation des ressources naturelles et la perte irréparable d'un patrimoine unique créé par des millions d'années d'évolution de la vie sur terre. Par ailleurs, leur création est le fruit des efforts consentis par les gouvernements à travers le monde pour conserver la biodiversité.(Coté, 2009).

En Côte d'Ivoire, les aires protégées forment un réseau de huit parcs nationaux et six réserves qui couvrent plus de six pour cent du territoire national. Elles regorgent de nombreuses potentialités d'ordres culturel, esthétique, économique, scientifique....malgré leurs avantages, elles ne sont pas totalement à l'abri de la dégradation des ressources naturelles qu'elles renferment. (UICN, 2008). En effet, l'exploitation agricole, le prélèvement des produits ligneux, la pêche, l'élevage, les feux de brousse, le braconnage sont de véritables menaces pour le maintien de l'équilibre biologique des espèces (UICN/BRAO, 2008).

Le Parc National de la Comoé (PNC) est inscrit sur la liste du patrimoine mondial de l'UNESCO depuis $1983^{41}$, du fait de sa grande richesse touristique et culturelle. Particulièrement, ilsubit les pressions du braconnage, des infiltrationsclandestines à des fins de cultures, les feux de brousse, la transhumance, les conflits politiques ainsi que des contraintes d'ordre institutionnel(UICN, 2008).Les menaces et pressions anthropiques qui s'effectuaientdélibérément par les populations pendant la période de crise

\footnotetext{
${ }^{41} \mathrm{https} / / /$ fr.unesco.org/news/site-du-parc-national-comoe-cote-ivoire-retire-liste-dupatrimoine-peril (consulté le 01 décembre 2018)
} 
sociopolitique de la Cote d'Ivoire sur le (PNC), en l'absence de surveillance, ont engendré des impacts potentiels, qui ont été à l'origine de son inscription sur la liste du patrimoine mondiale en péril de l'UNESCO en 2003. Il s'agissait notamment des troubles civils ; du déclin des populations de grands mammifères en raison d'une recrudescence de braconnage incontrôlé ; et de l'absence de mécanismes de gestion efficace. (Comité du Patrimoine Mondial, 2012). Le rapport des décisions de ladite session a mentionné comme menaces qui pèsent encore sur le Parc national de la Comoé. Il s'agit, principalement du: braconnage; de la divagation de bétail et de l'empiètement agricole. (Djafarou et Kalpers,2013).

Aujourd'hui, on note la présence permanente et opérationnelle du comité de gestion sur le terrain notamment à travers les activités de surveillance, de suivi écologique, d'aménagement et de sensibilisation. Ces efforts ont engendré des résultats probantsen 2017, qui ont valu le retrait du PNC de la liste du patrimoine mondial en péril par l'UNESCO (Rainfroy, 2017).Par ailleurs, de nombreux défis sont à relever; il s'agit entre autre de la préservation des services éco systémiques produits par le parc ainsi que la promotion du tourisme. N'empêche que les menaces et pressions anthropiques reconnus sur cette aire protégée existent toujours même si elles se font à faible échelle. Aussi, ces pressions sont-elles causées par les populations riveraines qui, le plus souvent enclavées, exploitent les ressources naturelles du PNC pour assurer leur survie.(Sissoko et Goh, 2010)

Cependant,au regard de la reprise effective des opérations du comité de gestion du PNC, une question fondamentale se pose, comment parvenir à conserver la biodiversité du parc sans toutefois priver les populations riveraines de leur survie? En d'autres termes, quelle est la problématique de gestion durable duPNC ? Le but visé en abordant cette étude est d'analyser les facteurs à l'origine de la problématique de gestion du PNC. Cet article rapporte les résultats d'une étude qui ne s'appuie que sur un seul objectif spécifique; celui d'identifier les types d'obstacles liés à la conservation de la biodiversité du PNC.

Cet objectif spécifique pourrait être éclairci autour de l'hypothèse selon laquelle une conjugaison de facteurs socioculturels serait à la base de la problématique de la conservation de la biodiversité du PNC.

Notre réflexion s'articule autour d'un plan qui part de la méthodologie de recherche à partir de laquelle nous effectuons une analyse et tirons des conclusions. 


\section{I-APPROCHE METHODOLOGIQUE}

\section{1-1- Site d'étude}

\section{1-1-1Brève historique du Parc National de la Comoé (PNC).}

C'est en 1926 que le colonisateur a défini les limites d'un refuge Nord qui devient par arrêté $\mathrm{N}^{\circ} 22$ du 3 Novembre 1942 la Réserve de faune de Bouna dont la partie occidentale bénéficie d'un simple statut de Réserve partielle.

En 1953, elle prend dans son ensemble le statut de Réserve total de faune avant d'être érigée en Parc National par décret $\mathrm{N}^{\circ} 68-81$ du 9 février 1968.Le PNC avait une superficie de 1150000 hectares. Par la suite le décret 77-116 du 25 février 1977 a retranché 850 hectares de sa surface au bénéfice de la ville de Téhini réduisant son étendu à 1149150 hectares. (N'guetia et Ehouman, 2012.)

Le Parc de la Comoé est inscrit sur la liste du patrimoine mondiale de l'UNESCO et désigné réserve de biosphère depuis 1983.

\section{1-1-2-Situation géographique et administrative du PNC.}

Situé au Nord-Est de la Côte d'Ivoire, le Parc National de la Comoé (PNC) est le plus vaste Parc d'Afrique de l'ouest.Il est situé entre les latitudes $8^{\circ} 30$ - $9^{\circ} 37$ Nord et les longitudes $3^{\circ} 07$ - $4^{\circ} 26$ Ouest, le Parc National de la Comoé (PNC) s'étend sur une superficie de 1149150 hectares forestières du domaine guinéen.IL s'étend sur les Préfectures de Téhini au Nord, de Bouna au Nord-Est, de Nassian au Sud et Sud-Est, de Dabakala au Sud-Ouest ainsi que de Kong au Nord-Ouest. IL doit son nom au fleuve Comoé qui le traverse du Nord au Sud. Le PNC, est limité à l'Est par la République du Ghana et la Volta noire; au Sud, par les Départements de Bondoukou et de Nassian;à l'Ouest, par les Départements deDabakala et Ferkessédougou ; au Nord, par les Départements de Doropo et de Téhini. IL s'étend sur huit Sous-préfectures qui sont : Bouna, Dabakala, Kong, Koutouba, Nassian, Sominassé, Téhini, et Tougbo. 


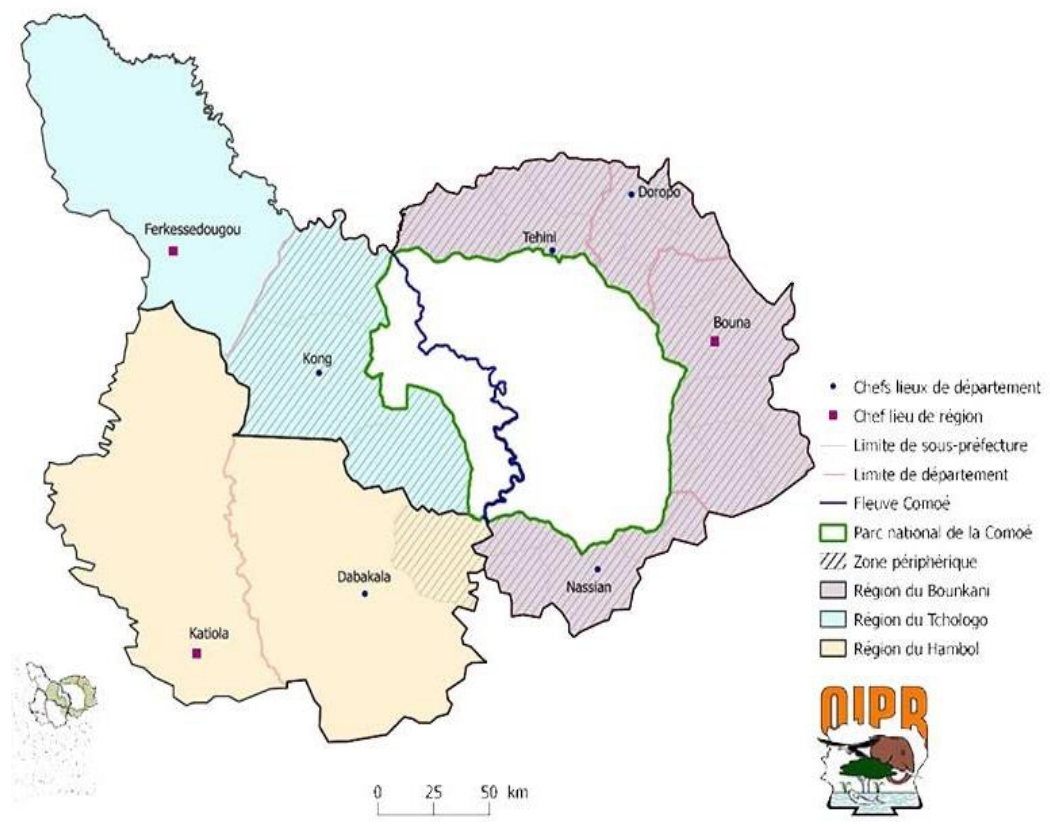

Figure 7 : Les, circonscriptions administratives du PNC en Côte d'Ivoire OIPR, Non daté, direction de zone Nord-Est (DZNE).

\section{1-2 Justification du choix du terrain d'enquête}

Le choix du terrain d'enquête s'est porté sur cinq villages riverains du PNC dont Kopkingué, Bania, Sépidouo, et Sialèdouo dans la sous-préfecture de Bouna et le village d'Ondéfidouodans la sous-préfecture d'Ondéfidouo. Etant dans l'impossibilité de couvrir tous les villages riverains du parc dans le cadre de notre étude, les critères qui ont prévalu au choix de ces cinq villages sont la forte démographie, le manque d'activités et d'échanges commerciaux. Ce choix à été fait, afin d'analyser les impacts des caractéristiques socioéconomiques et culturels du milieu d'enquête sur la conservation de la biodiversité du PNC.

\section{I-3 Population d'enquête}

La population d'enquête se compose:

-d'autorités traditionnelles telles que les chefs de villages et notables;

-de responsables d'associations villageoises (présidents de jeunes et présidentes de femmes).

-d'une autorité de l'Etat Monsieur, le Sous-préfet de Bouna.

-d'un responsable d'institution, le chef secteur à l'Office Ivoirien des Parcs et Réserves de Bouna (OIPR). 


\section{1-4 Caractéristiques des populations et activités socioéconomiques}

La population d'enquête est constituée de deux grands groupes ethniques qui sont les Koulango et les lobi.

\section{1-4-1Caractéristiques des populations \\ $\checkmark$ Les Koulango}

Ils ont pour ancêtres les Lorhons qui étaient les premiers occupants de la région depuis plus de 2000 ans. LesKoulango sont issus du groupe Voltaïque ou Gour. En leur qualité de premiers autochtones de la région, les Koulango demeurent les propriétaires coutumiers des terres du département de Bouna (Anonyme, 2012). A l'origine animistes, les Koulango et en particulier ceux du centre du royaume (Sous- préfecture de Bouna) sont aujourd hui dominés par la culture Malinké (Dioula) du fait de leur conversion à I'Islam. Ils sont situés à l'Est d'une ligne Nord Sud définie par la rivière Bawé, les monts Gorowi et le fleuve Comoé jusqu'à Téhini au Centre Nord du PNC; des environs de Bouna jusqu'à Doropo. (Anonyme, 2012).

\section{$\checkmark$ Les Lobi}

C'est la dernière communauté à arriver dans le royaume et par vagues successives. Cette importante migration vers le pays Koulango fera des Lobi au plan démographique, le groupe le plus important. Les peuples Lobi sont caractérisés par leur grande mobilité résidentielle à travers l'espace Volta Noire, Comoé et même au-delà. En conséquence, on distingue d’une part les Lobi ivoiriens installés dans le royaume depuis la période coloniale et d autre part les Lobi burkinabé et ghanéen dont le nombre est de plus en plus croissant depuis l'indépendance de la Côte d'Ivoire. Ils sont de grands éleveurs et agriculteurs. Bien que la cohabitation soit de manière générale pacifique avec les premières communautés sus citées, il n`est pas rare de constater quelques troubles liés aux problèmes fonciers mais aussi et surtout à la suite des conflits opposant les agriculteurs aux éleveurs. (Anonyme, 2012).

\section{1-4-2Activités socioéconomiques}

Les principales activités socioéconomiques au niveau de la zone d'étude sont l’agriculture, l’élevage et la chasse. Il y existe aussi l’artisanat et le commerce mais qui sont peu développés.

$\checkmark$ Agriculture

Elle représente la principale source de revenue des populations et occupe plus de $90 \%$ de la population active. Elle est dominée par les exploitations familiales de type traditionnel. C'est une agriculture itinérante utilisant les outils archaïques et les feux pour le défrichement des parcelles.Cette agriculture est dominée par les cultures vivrières telles que l'igname, le maïs, le mil, le sorgho, le riz de bas fond, le haricot et le néré. L'igname est la principale culture vivrière et la variété appelée «kponen » 
constitue le bien le plus prisé. Cette culture occupe la première place car, elle résiste naturellement aux irrégularités interannuelles de précipitations dans la région. La culture du coton dans la région fut un échec du fait des basses précipitations, par contre, celle de l'anacarde est en pleine expansion. L'agriculture dans la région ne se fait pas sans conséquences car les pratiques culturales se font de façon extensive et constituent une pression pour le PNC. Par ailleurs, il convient de signaler que de nombreux dégâts de cultures occasionnés par les éleveurs causent d'importants préjudices aux agriculteurs. (Anonyme,2012).

$\checkmark$ Elevage

Le Département de Bouna est une zone propice à l'élevage. Il se fait de manière traditionnelle. En effet, l'élevage de bovins et de petits ruminants constituent le second pilier de l'économie du Département. A côté des bovins nous notons les volailles, les ovins, les caprins et les porcins.

Par ailleurs, la région est soumise à des troupeaux conduits par des éleveurs peuhls transhumant des pays du Nord de la Côte d'Ivoire vers le Sud du pays. Aussi, de nombreux problèmes sont-ils enregistrés dans ce domaine d'activité. Les éleveurs sont confrontés aux difficultés liées à l'occupation anarchique des terroirs villageois par les agriculteurs qui étendent les superficies des cultures vivrières et de rentes et sur les pistes et barrages pastoraux. Les affrontements meurtriers surviennent très souvent entre agriculteurs et éleveurs suite aux dégâts de cultures.(Anonyme, 2012)

\section{$\checkmark$ Chasse}

Officiellement interdits par une décision présidentielle en 1974, la chasse et le commerce de gibiers se rangent aujourd'hui parmi les principales menaces de la biodiversité du PNC. Elle est menée de façon individuelle ou de façon collective par des autochtones (généralement les Lobis) ou les étrangers (généralement les Burkinabés et les Maliens). (Sissoko et Goh, 2010).

\section{$\checkmark$ Infrastructures économiques et commerce}

Les pistes qui mènent à Ondefidouo, Sepidouo et Sialedouo sont impraticables.

Le commerce n'est pas très développé dans notre zone d'étude. Seuls les villages de Sialedouo, Sépidouo et Ondéfidouo ont un marché. Kopkingué et Bania n'ont pas de marché. Le mauvais état des routes et l'absence de marché dans certains villages ne favorisent pas les échanges commerciaux par conséquent, l'exploitation des ressources biologiques devient pour certains villageois le moyen le plus facile pour gagner de l'argent.

\section{1-5 Technique d'échantillonnage}

Un échantillon de population à choix raisonné a pris en compte les catégories sociales ayant un lien avec le PNC dans les cinq villages enquêtés. 
L'échantillon comprend donc :

- Cinq (5) chefs de villages soit un (1) chef par village ;

- Vingt(20) notables soit quatre(4) notables par village ;

- Dix (10) responsables d'associations de villages dont une responsable d'association de femmes et un responsable d'association de jeunes par village

- un (1) responsable administratif, le Sous-préfet de Bouna ;

- un (1) responsable de service technique à l'administration, le chef secteur à l'OIPR de Bouna.

Soit un total de trente-sept (37) personnes composant notre échantillon.

\section{2-1-1Collecte de données}

\section{2-1-1-1Techniques et outils.}

Les outils de collecte de données telles que l'Observation directe, la documentation, l'interview semi directif (guide d'entretien) ainsi que le focus group ont été privilégiés.(KAKAI.. H 2008)

La documentation de notre étude se compose d'ouvrages, de rapports et d'articles relatifs aux fonctions des aires protégées, aux perceptions des usagers, aux pressions et impacts anthropiques sur ces sites. A partir d'investigations de terrain nous avons pu observer des indices liés aux pressions anthropiques notamment des pistes à l'intérieur du parc, des restaurants de viande de brousse. Des documents monographiques et cartographiques nous ont permis de décrire les caractéristiques socioculturelles des populations et de situer le parc par rapport à notre zone d'étude. Le guide d'entretien utilisé a été utile à l'analyse en profondeur des éléments recueillis, et a permis de récolter les témoignages et les interprétations des interlocuteurs en respectant leurs propres langages. Le focus group à été utile à recueillir des informations diverses riches et nuancées, compte tenu de la diversité des individus qui composait le groupe.

\section{2-2 Méthodes d'analyses}

La méthode qualitative a été privilégiée dans l'analyse des données d'enquêtes notamment à travers l'analyse de contenu.

\section{2-2-1Analyse de contenu}

Les données d'enquêtes, notamment les discours des participants recueillis par la technique de l'entrevue semi structurée ont fait l'objet d'une analyse de contenu (Wanlin, 2007). Le principe de cette méthode se situe dans la formation de catégories qui possèdent chacun un sens propre. L'on a donc regroupé et classé les discours selon les thèmes se référant à notrehypothèse de recherche. Ainsi, les discours des différents enquêtés ont pu être classé en trois thématiques; à savoir les facteurs d'ordre socioculturel, institutionnel et 
économique. Les propos des enquêtés «verbatims » sont transcrits entre guillemets et classés (sans l'aide d'un logiciel d'analyse de données).

\section{2-2-1-2 Analyse anthropobiologique selon la théorie de l'adaptation}

L'adaptation tire son origine de la théorie de l'évolution en biologie. Aujourd'hui son usage est transdisciplinaire. En anthropologie au sein de l'école américaine(Robson,1978) il est défini comme le processus par lequel les organismes ou les populations d'organismes effectuent des ajustements biologiques ou comportementaux qui facilitent et assurent leur succès reproducteur et donc leur survie dans leur environnement.

En bioanthropologie, discipline dans laquelle s'est élaborée cet article, l'utilisation de la théorie de l'adaptation passe par l'étude de l'évolution des cultures humaines ainsi que les changements culturels et biologiques qui se sont déroulés au fil du temps. En analysant les retombées positives ou négatives de ces changements, cette théorie s'intéresse à la manière dont les individus ou les groupes s'adaptent à leurs environnements. Notre analyse a donc pris en compte les aspects culturels qui ont donc subit des modifications, restrictions, ou qui ont totalement disparus du fait de l'érection du domaine forestier en parc national. De plus, le comportement des populations face aux ressources naturelles en dépit de l'interdiction formelle de les exploiter est analysé sous l'angle de l'adaptation de ces populations à leurs survies.

Il convient avant de présenter les résultats de clarifier les concepts clés à l'étude pour une meilleure compréhension de l'article.

\section{2-3 Clarification de concept}

La problématique c'est l'ensemble des problèmes ou obstacles qui paralysent la gestion efficace du PNC.

Du latin gestio, le concept de gestion se réfère à l'action et à l'effet de gérer ou d'administrer. Gérer, c'est prendre des mesures conduisant à la réalisation d'une affaire ou d'un souhait quelconque. D'autre part, administrer, c'est gouverner, diriger, ordonner ou organiser.

Le terme gestion concerne donc l'ensemble des procédures effectuées pour résoudre un problème ou réaliser un projet. La gestion est également la direction ou l'administration d'une entreprise ou d'une affaire.

Il existe plusieurs types de gestion. La gestion sociale, à titre d'exemple, consiste à construire divers espaces d'interaction sociale.

La gestion environnementale est l'ensemble des mesures ou procédures dédiées au système environnemental fondé sur le développement durable. Elle se présente comme la stratégie par le biais de laquelle sont 
organisées les activités humaines nuisant à l'environnement, dans le but de parvenir à une meilleure qualité de vie. ${ }^{42}$

La gestion des aires protégées se réfère aux différentes procédures et investigations menées dans le but de la préservation et de la protection des systèmes écologiques tout en tenant compte des décisions des populations, pour un développement durable.

Une aire protégée est définie comme «Un espace géographique clairement défini, reconnu, consacré et géré, par tout moyen efficace, juridique ou autre, afin d'assurer à long terme la conservation de la nature ainsi que les services écosystémiques et les valeurs culturelles qui lui sont associés » (Dudley, 2008).

Un parc national désigne une aire placée sous le contrôle de l'Etat et dont les limites ne peuvent être changées, ni aucune partie aliénée, sauf par l'autorité législative compétente ;

- exclusivement destinée à la propagation, la protection, la conservation et l'aménagement de la végétation et les populations d'animaux sauvages, ainsi qu'à la protection des sites, des paysages, ou des formations géologiques d'une valeur scientifique ou esthétique particulière, dans l'intérêt et pour la récréation du public ;

- dans laquelle l'abattage, la chasse, la capture d'animaux et la destruction ou la collecte des plantes sont interdits, sauf pour des raisons scientifiques ou pour les besoins de l'aménagement et à condition que de telles opérations aient lieu sous la direction et le contrôle de l'autorité compétente ;

- comportant tout milieu aquatique auquel s'appliquent toutes ou l'une quelconque des dispositions des alinéas 1 et 3 de la présente définition (Loi $\mathrm{N}$ 2002-102 du 11 février 2002 relative à la création à la gestion et au financement des parcs nationaux et des réserves naturelles.

La biodiversitéou diversité biologique est définie par la convention sur la diversité biologique ${ }^{43}$ comme la «variabilité des organismes vivants de toute origine y compris, entre autres, les écosystèmes terrestres, marins et autres écosystèmes aquatiques et les complexes écologiques dont ils font partie ; cela comprend la diversité au sein des espèces et entre espèces ainsi que celle des écosystèmes ».

Ainsi, la notion de «diversité biologique» englobe-t-elle toute la flore, la faune et les microorganismes terrestres et aquatiques ainsi que les écosystèmes au sein desquels ces ressources apparaissent et survivent.

La diversité biologique comprend, d'une part, les ressources génétiques contenues dans les races et les espèces animales et végétales et, d'autre part, les communautés écologiques au sein desquelles sont créées les

\footnotetext{
${ }^{42} \mathrm{http}: / /$ lesdefinitions.fr/gestion (consulté le 01 décembre 2018, 11h05)

${ }^{43} \mathrm{http}: / /$ www.cbd.int/(consulté le 01 décembre 2018, 11h20)
} 
conditions qui entretiennent la vie de toutes les populations. La préservation de la diversité biologique implique la conservation des ressources génétiques, des espèces animales et végétales et le maintien de toutes les composantes des écosystèmes.(Triplet.P et Eloundou.L, 2009).

Elle est donc la variété des diverses formes de vie ainsi que les différents habitats ou écosystèmes dans lesquels ces vies se développent.

La conservation de la biodiversitéest lagestion des interactions humaines avec les gènes, les espèces et les écosystèmes afin de fournir le maximum d'avantagesà la génération actuelle tout en maintenant leurs potentiels pour satisfaire les besoins et les aspirations des générationsfutures ; la notion également comprend la sauvegarde et l'étude de l'utilisation de la biodiversité. Triplet, 2009.

La survie est le fait de vivre après un évènement particulier... d'essayer de vivre dans des conditions difficiles et sans en avoir les moyens ${ }^{44}$.

Les techniques de survie sont des compétences qui permettent aux personnes de rester en vie si elles se retrouvent isolées au milieu de la nature. Ces techniques leur permettent de se nourrir, de se protéger des intempéries et de guérir d'éventuelles blessures légères. Dans le contexte de notre étude, la survie des populations riveraines est le fait d'assurer leurs subsistances en dépit de l'interdiction formelle d'accéder au PNC pour d'éventuels prélèvements ou exploitations de ressources naturelles.

\section{II- RESULTATS}

1- 1 - Facteurs favorisant l'exploitation des ressources biologiques dans le PNC

Les facteurs favorisant l'exploitation des ressources biologiques dans le PNC sont d'ordres socioculturels, économiques et institutionnels.

\section{1-1-1- Facteurs socioculturels}

\section{1-1-1-1- Perceptiondes populations}

Le PNC étant le patrimoine de l'Etat, aucune intrusion humaine sans autorisation préalable de l'autorité compétente n'est possible. Cette situation provoque parfois chez les populations une amertume et des ressentiments. Les populations se sentent marginalisées dans le projet de conservation de cette aire protégée et surtout elles ont le sentiment d'avoir été expulsées de leur terre. Ce qui les pousse d'ailleurs à outrepasser les lois d'interdictions et à s'introduire dans ce domaine pour défendre un droit qui à leurs yeux serait violé. Nous analysons ce sentiment à travers des propos suivants: "le mauvais état de conservation du parc s'explique par la présence des hommes dans le parc. C'était le domaine de nos grands-parents et l'Etat nous a délogé .Si les

\footnotetext{
${ }^{44}$ http://lesdefinitions.fr/survie(consulté le 01 décembre 2018, 11h40)
} 
gens rentrent dans le parc c'est qu'ils y trouvent leur intérêt !Propos d'une autorité coutumière a Kopkingué.

Certaines personnes n'ont aucune idée du profit que peut leur procurer le PNC.A cet effet voici ce que pensent nos enquêtés de la proximité de ce site.

"Le parc est pour le gouvernement je ne sais pas si cela m'arrange ou pas! »Discours recueillis auprès d'une autorité coutumière à Ondéfidouo.

"Les touristes venaient faire des cadeaux à nos enfants avant la crise hormis cela nous ne voyons pas notre profit aujourd'hui .On nous dit qu'on a du profit mais nous ne voyons rien. » Déclarationd'une autorité coutumière à Bania

"La proximité du parc ne nous gêne pas mais si on pouvait avoir une parcelle du parc pour la culture, cela nous ferait plaisir parce qu'avec la culture de l'anacarde on a presque plus de terre et elles ne sont plus fertiles. » Ce sont lespropos d'une autorité coutumière à Ondéfidouo.

Certains enquêtés pensent même qu'on devrait attribuer la tutelle du parc aux populations.

A ce propos, une autorité coutumière à Sialèdouo nous révèle ceci : Pour que le braconnage prenne fin, il faut libérer le Parc pour que les populations aillent librement chasser et y faire leur champ!».

Les différentes représentations que les populations se font du parc nous laissent croire que pour elles, le parc reste la seule ressource naturelle pouvant leur permettre d'avoir une source de revenus. Elles s'adonnent ainsi à l'exploitation des ressources biologiques de cette aire.

Les propos de la présidente des femmes à Kopkingué illustre quelque peu notre analyse : " Avant la crise, le parc nous permettait de prélever des noix de karité. Nous les vendions et cela nous permettait de scolariser nos enfants mais aujourd'hui, les autorités nous interdisent de rentrer dans le parc. La situation est difficile pour nous. »

\section{1-1-1--2-Pratiques cultuelles.}

En Afrique, l'origine du culte se rapporte à la nature. Les pratiques cultuelles ont toujours été rattachées à l'environnement. Par ailleurs, la forêt, les cours d'eaux, les animaux, les montagnes etc., de par la beauté qu'ilsrévèlent et surtout de leurs forces, incarnent un pouvoir surnaturel pour lequel un culte leur est adressé. Ainsi, certains cultes sont adressésà la rivière, aux montagnes et même à certains animaux. De même certaines parties du corps des animaux entrent dans la fabrication de remèdes contre certaines maladies, pour acquérir soit la guérison soit certains pouvoirs mystiques... Avant le néolithique, l'homme se conçoit comme partie intégrante de son environnementpuis, en domestiquant la nature au néolithique, il s'en détache et forme une entité distincte qui interagit avec elle et c'est de là qu'il acquiert 
son pouvoir sur la nature, sa capacité à la transformer pour assurer sa propre survie.

Chez les Lobi et les Koulango la pratique de la chasse, est la matérialisation de leur culture.A travers la chasse, certains hommes surtout les Lobi, se distinguent d'autres en termes de puissance; il existe des cérémonies d'initiation à la chasse qui leur confèrent un statut de pouvoir. En effet les initiés à la chasse possèdent des talismans (des objets qui servent de protection en cas de dangers); ils sont infaillibles grâce aux pouvoirs que leur confère le fétiche. La puissance de ces hommes se perçoit donc à travers la capacité à chasser les gros animaux (les buffles) et surtout les plus féroces. La tête d'un gros animal devient un palmarès pour les chasseurs. Par ailleurs, la plupart des Lobi composant notre localité d'enquête sont des animistes. Leur culte nécessite souvent des cérémonies de sacrifices d'animaux sauvages. En effet, les populations s'adonnentà la chassepour pouvoir demander l'aide dans les champs car chez les Lobi on demande de l'aide avec de la viande de brousse. Elles y vont aussi lorsque leur fétiche réclame un animal sauvage pour des rituels.

\section{1-1-2 Facteurs économiques}

Plusieurs facteurs constituent des contraintes au projet de conservation du PNC. Ils'agit notamment de l'extrême précarité du milieu matérialisé par l'impraticabilité des routes, l'absence d'électricité et de marché dans la plupart des villages riverains du PNC.

En effet, sur les cinq villages enquêtés, seulement deux sont contigus au parc et les voies d'accès aux trois autres villages sont impraticables.

La présidente des femmes à Sialèdouo nous révèle ceci : "la voie d'accès à notre village est très abimée ce qui nous crée beaucoup de problèmes avec nos marchandises. Les conducteurs de camions refusent de venir chercher nos marchandises et aussi de nous faire des livraisons ce n'est pas leur faute car très souvent il y'a des camions qui sont embourbés avec des marchandises!»

L'absence de marché et d'électricité dans la plupart des villages riverains est un handicap au développementd'une industrie de commerce.

La pauvreté dans les villages riverains du PNC est une cause majeure de la dégradation des ressources naturelles. En effet, le mauvais état des pistes ne favorise pas les échanges commerciaux et l'absence d'électricité associée à la mévente des produits agricoles rend le milieu précaire. Les populations n'ayant pas d'autres choix s'adonnent ainsi au braconnage et au commerce de la viande de brousse. 


\section{1-1-3 Facteurs institutionnels}

L'OIPR dans la lutte contre la dégradation de la biodiversité dans le PNC a prévu deux grandes actions à savoir les missions de patrouilles et la sensibilisation des populations riveraines. Le responsable de l'OIPR nous explique ceci : "...Nous avons plusieurs équipes; chaque mois, chaque équipe passe un temps dans le parc à la recherche de tous ceux qui sont en situation illicite. A côté de cela, nous avons la communication afin que certains comprennent et nous assiste dans le domaine de la surveillance. „Cependant quelques failles paralysent la gestion efficace de la biodiversité du parc.

\section{1-1-3-1 Missions de patrouille}

Les missions de patrouilles sont effectives et régulières dans le PNC. Les chasseurs et les autres enquêtés nous ont révélés des problèmes.

"Des problèmes on en connait plein. Des personnes sont appréhendées maltraitées et paient des amendes souvent de quatre cents cinquante mille, souvent de sept cents mille pour être libre... », sont les propos du chef du village de Bania.

"Des personnes ont été maintes fois surprises dans le PNC, vers Kafolo, un combat a été mené entre braconniers et agents des Eaux et Forêts l'année dernière. », (Propos de la présidente des femmes à Sialèdouo).

"Dans notre village, des personnes ont déjà été appréhendées aussi, il ya des personnes des villages voisins tels que Sépidouo etOndéfidouo. Lorsqu'elles sont prises dans le parc on les maltraite, on les frappe, on confisque leur fusil et elles sont obligées de vendre leur bien pour être libre .Souvent il ya des personnes qui vendent leur plantation d'anacarde, leus parents sont obligés de les aider à payer leur amende qui peut coûter même sept cents mille francs. C'est vraiment malheureux car cela a appauvri complètement des familles ici. »(Propos du chef du village de Sialèdouo).

Malgré l'effectivité des missions de patrouilles, on note des difficultés d'ordres matériel, infrastructurel et humain. Il ya le manque de matériels roulants, le nombre insuffisant d'agents des eaux et forêts pour assurer les patrouilles, manque de moyens de communication, manque d'armes pour la sécurité des agents des Eaux et Forêts, déficit d'infrastructures routières, manque d'outils et de spécialistes en communication.

Le responsable à l'OIPR de Bouna n'a pas manqué de le signifier à travers ses propos:

«Nous n'avons pas de matériels roulants et c'est vraiment dommage. Nous n'avons pas beaucoup d'armes alors que les braconniers sont armés .Les pistes ne sont pas aménagées et les braconniers le savent; ils savent pertinemment qu'il ya des endroits où les agents des forces de l'ordre ne peuvent accéder à cause de l'état des pistes. En matière de communication, 
nous avons un problème d'outils. Nous avons un déficit d'animateurs ruraux pour la sensibilisation

\section{1-1-3-2 Sensibilisation}

La sensibilisation est le moyen le plus probable pour toucher les populations, les informer et les éduquer à la protection de la biodiversité du PNC. En effet, devant la fragilisation du PNCdue aux multiples agressions, il convient d'envisager plusieurs possibilités qui pourraient renverser la tendance. Les parcs nationaux loin d'être des sites d'empiétements de ressources naturelles peuvent servir à la sensibilisation et à l'éducation car ayant un but touristique et de loisir.Il incombe donc de connaitre tous les acteurs impliqués dans la conservation afin de les informer et les éduquer à la protection du PNC.

Il semble de ce fait que le projet de sensibilisation n'ait pas encore atteint les objectifs souhaités, car le responsable de L'OIPR le mentionne en ces termes : "l'implication des populations riveraines dans la gestion du parc n'est pas totalement acquise. Pourtant, il faut que les deux acteurs au moins c'est-à-dire l'OIPR et les populations riveraines parmi tant d'autres $s$ 'associent à la gestion durable du parc. Il faut que les populations trouvent des solutions à partir de leur implication dans la gestion durable du parc. »

La concertation et les partenariats des pouvoirs locaux et des populations doit être de mise de façon permanente afin d'avoir des résultats partagés à long terme. La prise de conscience ne sera acquise qu'après une sensibilisation plus grande sur certains enjeux irréversibles de l'environnement et doit s'étendre impérativement sur les plus jeunes afin que ceux-ci soient empeignés des réalités et des conséquences environnementales en tant qu'acteurs de demain afin de développer et faciliter des aptitudes plus responsables à la conservation de l'environnement. Il faut surtout que les populationsprennent conscience de leur mode de vie et constatent par la même occasion l'amélioration de leursconditions de vie à travers leurs implications dans le projet de sensibilisation.

\section{III- DISCUSSION}

Les pressions sur les aires protégées par les populations riveraines peuvent s'expliquer en s'appuyant sur l'historique de leur installation. En effet, la perception des populations de l'aire protégée est fortement liée à l'historique et aux conditions de classement du PNC. Lors du classement du $\mathrm{PNC}$ en un domaine permanent de l'Etat ; les populations riveraines ont perdu tous les droits et usages sur cet espace. Certains villages ont même été déplacés lors du classement. Cette rupture d'avec leur patrimoine ne s'est faite sans conséquences. Les populations ont dû abandonner leurs champs, leurs pratiques culturelles qui pour la plupart impliquaient l'utilisation des 
ressources naturelles du PNC. Ces populations mécontentes et frustrées ont le sentiment d'avoir été spoliées de leurs biens. Etant pour la plupart dans des zones enclavées, elles s'adonnent à l'exploitation des ressources naturelles en dépit de l'interdiction. C'est la raison pour laquelle (Medou,2002)affirme que l'expropriation des populations dans le but du classement des aires protégées est un danger pour la conservation en ce sens que les populations victimes d'expropriation de terres se vengent en créant des conflits et en exploitant les ressources à des fins commerciales.

Aujourd'hui avec la démographie galopante, ces pressions se font de plus en plus fortes et constituent une véritable problématique pour les gestionnaires de l'aire. En effet, les difficultés liées à la gestion des aires protégées font souvent intervenir la problématique de tutelle. Selon (Sournia, 1998) auparavant, le classement des aires protégées intervenait dans des régions peu peuplées et pour des raisons soit historiques,(zones tampons entre plusieurs peuples ou terrains de conflits locaux) soit écologiques (présence de mouches tsé-tsé, pauvreté naturelle des sols, absence d'eau...Ainsi, ces zones non perturbées étaient de ce fait relativement riches en faune et constituaient des sites de classement et de protection idéaux. Seulement depuis une vingtaine d'années les critères qui ont rendu possible ces classements ont radicalement changé.

L'augmentation de la population a occasionné une recherche généralisée des nouvelles terres à mettre en valeur. Depuis les années soixantedix, le phénomène a été accentué par les brutales modifications climatiques créant un exode massif des populations en direction du Sud. Ces migrants considéraient les aires protégées comme des futurs fronts pionniers potentiellement riches en eau, en sols en pâturages...Ils exercent désormais des droits sur ces zones jadis conservées. Ce constat constitue pour le gouvernement et les organismes de protection des ressources naturelles l'un des plus grands défit de ce siècle en matière de conservation des écosystèmes, de politiques d'aménagement de territoires et de gestion de ressources naturelles.

Dans le PNC, le recours à l'exploitation des ressources naturelles apparait comme un moyen pour les populations de survivre, de s'adapter aux modifications et aux changements de leurs milieux de vie, perturbés par la démographie galopante et les conséquences du changement climatique, ponctués par la pauvreté. De plus, si elles sont interdites de pratiques diverses (agricoles, chasse, etc.), elles sont emmenés à enfreindre les interdictions pour leur survie. L'absence d'activités de substitution peut être source de conflits avec l'autorité de conservation. Cette situation est également relevé par Jordan (2014) qui affirme que « les activités communautaires si elles sont interdites dans la zone tampon ....conduit aux pratiques de diverses exactions contre 
[une] aire protégée, du fait que la population s'attaque ouvertement aux ressources tels que, la disparition des ressources animales et végétales du parc.

Outre la perception des populations comme obstacle à la conservation, nous soulignons les facteurs économiques et institutionnels. Il est important de noter que les problèmes de développement, la pauvreté, la course aux richesses, les intérêts politiques font très souvent oublier à l'homme qu'il est au centre de la protection de l'environnement.Malheureusement, à la suite des pressions anthropiques nous assistons au déséquilibre voir à la disparition des écosystèmes. Selon (Sournia, 1998), les causes de la raréfaction des animaux et des écosystèmes sont les mêmes en Afrique. Il s'agit de la destruction des habitats, des mises en cultures intempestives, de l'irruption des cultures inadaptées, et du braconnage incontrôlé... Concernant la Côte d'Ivoire, (UICN/BRAO, 2008), aborde la question dans la même logique en précisant que les aires protégées sont soumises à de fortes pressions qui sont les activités agricoles, l'exploitation forestière, la pêche, les feux de brousse incontrôlés et quelques pressions spécifiques telles que l'orpaillage, les pressions foncières, la pollution et le braconnage. Ces problèmes sont généralement engendrés par la faible application de la loi, le manque d'infrastructures et d'équipements pour la gestion des aires protégées et l'instabilité politique. Selon l'étude, dans le parc national de la Comoé, un braconnage intensif aurait eu lieu dans la partie du parc qui n'était pas surveillée par L'OIPR (ex zone de confiance et ex zone sous contrôle des forces nouvelles). Nous notons que les obstacles à la conservation des aires protégées sont aussi dus à des problèmes d'économie et de tutelle. (Sournia, 1998) renchérit sur cet aspect en mentionnant que la faune, les parcs nationaux, les réserves naturelles, sont des thèmes et sujets qui ont peu de place dans des ouvrages traitant des aspects du développement économique sur le continent Africain à l'exception de quelques organisations spécialisées telles que ONU-Environnement ${ }^{45}$ (Ex-PNUE $\left.{ }^{46}\right), \mathrm{UNESCO}^{47}$, $\mathrm{UICN}^{48}, \mathrm{WWF}^{49} \ldots$ Toutefois, l'UE ${ }^{50}$, la banque mondiale, les coopérations suisses, allemandes voir françaises et bien d'autres commencent à manifester un réel intérêt au financement de ces sites. Il est important de ne pas négliger un aspect tout aussi important entrainant les facteurs institutionnels et socioculturels. Il s'agit de l'inefficacité des lois environnementales.

En matière de conservation des aires protégées, l'inefficacité des normes s'identifie par des raisons valables pour toutes les autres normes

\footnotetext{
${ }^{45}$ ONU-Environnement Organisation des Nations Unies pour l'Environnement

46 PNUE: Programme des Nations unies pour l'environnement (aujourd'hui, ONUEnvironnement)

${ }^{47}$ UNESCO : Organisation des Nations unies pour l'éducation, la science et la culture

${ }^{48}$ UICN : Union internationale pour la conservation de la nature

${ }^{49} \mathrm{WWF}$ : Fonds mondial pour la nature

${ }^{50} \mathrm{UE}$ : Union Européen
} 
environnementales en Côte d'Ivoire et ailleurs dans certains pays Africains. Au Burkina Faso, pays transfrontalier à la Côte d'Ivoire, par exemple, les principales causes de l'inefficacité de la législation nationale en matière de gestion des aires protégées sont les causes institutionnelles, et socioéconomiques. En ce qui concernent les causes institutionnelles, on note l'insuffisance en nombre d'effectif pour couvrir l'ensemble du territoire national et les rares services qui existent se trouvent souvent dépourvus des outils élémentaires de travail (véhicules et autres équipements informatiques) et des moyens de fonctionnement (carburant, fournitures de bureau), indispensables à l'accomplissement de leur mission (UICN, 2010). Quant aux causes économiques, on note la pauvreté, qui entraine parfois la nécessité pour certains ménages de prélevercertaines ressources naturelles alors même que celles-ci peuvent faire l'objet d'une protection particulière dans le cadre des aires protégées. (UICN, 2010). Cette situation peut être illustrée au Burkina Faso notamment par la consommation du bois de feu par les ménages, ce qui entraîne des conséquences graves à long terme sur le couvert végétal, ou encore par la pratique de l'agriculture extensive ou de l'élevage transhumant... Il convient toutefois de rappeler qu'il s'agit là d'une consommation des ressources naturelles dépassant les droits d'usage reconnus par la loi aux communautés locales. (UICN,2010).

Considérant les arguments sus cités, nous constatons que les principaux problèmes de conservation des aires protégées en Afrique et même en Côte d'Ivoire s'identifient par le manque de financement et la faiblesse institutionnelle. Il incombe donc au regard des besoins de la nouvelle génération de reconsidérer les mécanismes de gestion. A cet effet, il est important d'adopter une vision nouvelle qui est celle d'un développement économique au-delà de la gestion purement conservatoire de l'environnement. Une nouvelle gestion économique dans laquelle les populations riveraines auront des droits réels est primordiale.

$\mathrm{Au}$ regard de la nouvelle gestion participative des acteurs dans la conservation des aires protégées, plusieurs études ont été élaborées dans le souci d'une amélioration de la gestion de ces sites. Dans cette logique, (UICNPAPACO, 2012)élabore les outils d'appui de décisions pour la conservation de la biodiversité des aires protégées d'Afrique compte tenu des fortes pressions qui entravent leurs gestions. Il s'agit du renforcement des compétences de managements des gestionnaires des aires protégées et leurs poids dans les décisions ; la reforme de ces aires et l'application des lois existantes ; la valorisation de ces sites et la promotion de leur intérêt auprès des bénéficiaires directs et indirects.

Pour résoudre efficacement le problème de la dégradation des ressources naturelles dans le PNC, il est donc nécessaire d'adopter la nouvelle vision participative entre l'Etat et les populations riveraines. Mais nous 
pensons qu'il est primordial de comprendre les besoins des populations. En d'autres termes, il faut étudier la situation socioéconomique des populations riveraines, analyser les critères qui pourraient constituer un obstacle à la conservation du PNC et veiller à les résoudre. C'est ainsi que dans le même ordre d'idée (Sissoko et Goh, 2010) en analysant la situation socioéconomique des riverains du parc national de la Comoé,ont confirmé qu'elle est un facteur explicatif de la dégradation avancée des ressources naturelles du PNC.

Pour finir, nous disons que la gestion durable des ressources naturelles du PNC n'est possible que dans la conciliation des besoins économiques des populations riveraines d'une part et la préservation de la biodiversité d'autre part, ce qui n'est d'ailleurs pas chose aisée; En effet, la privation, l'interdiction formelle d'accéder aux ressources biologiques du PNCconstitue pour les populations riveraines un manque à gagner.C'est pourquoi, (Triplet et Vermeulen, 2009), dans leur analyse sur la question du double enjeu de préservation de la biodiversité et de la survie des populations stipulent que les prélèvements de la flore ou de la faune sont réglementairement interdits dans la plupartdes aires protégées, et plus particulièrement dans les parcs nationaux, ce qui interdit le pâturage,l'utilisation des végétaux pour la pharmacopée, mais également le ramassage du bois de chauffe, la collecte de miel, de racines, de fruits. Cependant, de nombreuses ressources de collecte constituent en période de soudure alimentaire un complément alimentaire indispensable pour les populations locales.En outre, ces auteurs révèlent que certaines ressources recouvrent pour les populations des valeurs symboliques importantes dans les rituels. Ils préconisent, si l'occasion ait donné aux législateurs de déroger certaines règles, que les populations puissent faire des prélèvements de bois morts et d'autres produits forestiers sans toutefois que cela ne constitue une source d'appauvrissement de la ou des espèces végétales concernées. Dans tous les cas, le double enjeu demeure et mérite d'être encore analysé aux plus hautes instances pour un développement durable.

\section{CONCLUSION}

La problématique de la gestion de la biodiversité se résume en Côte d'Ivoire et plus particulièrement dans le PNC à concilier, d'une part, les besoins de développement socio-économique des sociétés et, d'autre part, la nécessité de préserver la diversité biologique. Le parc national de la Comoé fait l'objet de plusieurs pressions anthropiques.La faune ainsi que les autres ressources biologiques ont été décimée. L'on a pu confirmer à travers cette étude que, une conjugaison de facteurs tels que la précarité du milieu, les pratiques socioculturelles des populations riveraines et les contraintes d'ordre institutionnel sont à la base de la dégradation des ressources naturelles dans le PNC. Notre hypothèse est ainsi vérifiée. 
Face aux difficultés des populations et de l'OIPR qui paralysent la gestion efficace de ce patrimoine mondial, il convient de relever le défi notamment chez les populations en luttant contre la pauvreté, en créant des activités génératrices de revenu dans l'ensemble des villages riverains, en les associant à tous les projets et en privilégiant les bénéfices équitables déroulants de ces projets. Réformer la gestion de l'OIPR par un plan d'aménagement, le réaménagement des pistes intérieures du parc, des armes pour la sécurité des Agents des Eaux et Forêts, du matériel roulant et des experts en communication rurale.

Pour lutter contre la dégradation des ressources naturelles dans le PNC, il est nécessaire de privilégier la collaboration de tous les acteurs susceptibles d'avoir un impact sur les aires protégées et rechercher desfinancements à long terme.

\section{References:}

1. Anonyme, 2012. Monographie de Bouna.http://news.abidjan.net/h/445521.html_consulté le 01 décembre 2018).

2. Comité du Patrimoine mondial, 2012. Décision 36 COM 7A.2. Parc national de la Comoé. 36è session, Saint-Pétersbourg, Russie.

3. Coté. V, 2009. La prise en compte des populations locales dans la mise en place d'aires protégées : études de cas au Guatemala et au Maroc. Universitéde Sherbrooke, Québec.85p.

4. Dudley, N. (2008). Lignes directrices pour l'application des catégories de gestion aux aires protégées. Gland, Suisse : UICN. $\mathrm{x}+96 \mathrm{pp}$.

5. JORDAN, L.A. (2014).Problématique de la gestion de la zone tampon du parc national de Virunga et son impact sur les conflits parc-population. Institut Supérieur de Technique de Développement de Mulungu-Mémoire de Licence, https://www.memoireonline.com/10/17/10118/Problematique-de-lagestion-de-la-zone-tampon-du-parc-national-de-Virunga-et-sonimpact-sur-les-con.html, consulté le 10 décembre 2018.

6. Lauginie, F.2007. Conservation de la nature et aires protégées en Côted'Ivoire,Abidjan, NEI/Hachette et Afrique Nature International 668 $\mathrm{p}$

7. Medou.C. 2002 "Les aires protégées en Afrique: perspectives pour leur conservation », VertigO- la revue électronique en science de l'environnement(En ligne).Volume 3 Numéro 1/ avril 2002, mis en ligne le 01 avril 2002, consulté le 27mai2017.

8. N'gettia A et Ehouman P. 2012: le braconnage dans une aire protégée : cas du parc national de la Comoé. Rapport de stage OIPR Bouna. 
9. OIPR.2008. programmes cadres de gestion des aires protégées. PARCCI évaluation d'Impact environnemental et Social 83p.

10. Rainfroy. C, 2017. Côte d'Ivoire: le parc national de la Comoé n'est plus en péril, selon l'UNESCO. Jeune Afrique à Abidjan, Repéré à https://www.jeuneafrique.com/454042/societe/cote-divoire-parcnational-de-comoe-retire-de-liste-patrimoine-peril/consulté le 6 décembre 2018.

11. République de Côte d'Ivoire. 2002. Loi n²002-102 du 11 février 2002 relative à la création, à la gestion et au financement des parcs nationaux et des réserves naturelles, Abidjan: Assemblée Nationale de la Côte d'Ivoire.

12. Robson, E., 1978. Utilisation $d u$ concept d'adaptation en anthropologie,social, science, information, 17, 2, 279-335.

13. Sissoko, A. \& Goh, D., 2010 Situation socioéconomique des riverains $d u$ Parc national de la Comoé et leur interaction avec le parc. 88 pp. Abidjan.

14. Sournia.G 1998 : les aires protégées d'Afrique francophone Paris Edition Jean-pierre de Monza 40, rue marbeuf $75008272 P$.

15. Tiomoko, D et Kalpers, J. 2013 Mission de suivi réactif au Parc national de la Comoé Côte d'Ivoire rapport de mission UNESCO PARIS.

16. Triplet. P. 2009. Manuel de gestion des aires protégées d'Afrique francophone. Awely, Paris, pp.1215, <hal-00669157>.

17. Triplet. $\mathrm{P}$ et Vermeulen.C ,2009. Autoriser ou non les prélèvements des produits naturels sur les aires protégéesdansTriplet. P. Manuel de gestion des aires protégées d'Afrique francophone. Awely, Paris, pp. 1215, <hal-00669157>.

18. UICN /BRAO. 2008 : Evaluation de l'efficacité des aires protégées ; parcs et réserves de Côte d'Ivoire, Ouagadougou. 43 pp.

19. UICN/PACO, 2010.Évaluation juridique et institutionnelle pour la mise en place des conditions d'amélioration de la gestion des aires protégées d'Afrique de l'Ouest. Ouagadougou, BF: UICN/PACO.

20. UICN-PAPACO. 2012. Renforcer la conservation des aires protégées d'Afrique...Synthèse de la rencontre de Weotenga; Ouagadougou, janvier2012.

21. UNESCO, 2017. Le site du Parc national de la Comoé (Côte d'Ivoire) retiré de la Liste du patrimoine en péril, https://fr.unesco.org/news/sitedu-parc-national-comoe-cote-ivoire-retire-liste-du-patrimoine-peril (consulté le 01 décembre 2018)

22. Wanlin.P, 2007. L'analyse de contenu comme méthode d'analyse qualitative d'entretiens : une comparaison entre les traitements manuels et l'utilisationde logiciels. Université de Luxemboug recherches qualitatives - Hors Série - numéro 3 ;ISSN 1715-8702. 